In this special case the stability condition is

if the bed is frozen and

$$
\lambda_{m}=-\left[\frac{(2 m+\mathrm{I})}{2 h} \pi\right]^{2}+\frac{a}{K}<0 \quad m=0, \mathrm{I}, 2, \ldots
$$

$$
\lambda_{m}=-\left(\frac{m \pi}{h}\right)^{2}+\frac{a}{K}<0 \quad m=\mathrm{I}, 2,3, \ldots
$$

if it is at the melting point. The eigenvalue associated with the smallest value of $m$ is the least stable so the maximum stable thickness is thus $h=\frac{1}{2} \pi(a / K)^{\frac{1}{2}}$ if the bed is frozen or $h=\pi(a / K)^{\frac{1}{2}}$ if it is at the melting point. For typical flow-law parameters these depths are around $250 \mathrm{~m}$ and $500 \mathrm{~m}$ respectively. The eigenvalues are related in a simple way to the growth or decay rates of the eigenfunctions: $\left(\kappa \lambda_{m}\right)^{-1}$ is the time constant for the $m$ th eigenfunction.

Depth-dependent stress, temperature, and advection have a marked effect on stability. A slab in which stress and temperature increase to values $\sigma_{\mathrm{B}}$ and $T_{\mathrm{B}}$ at the bed is considerably more stable than a slab held at constant stress $\sigma_{\mathrm{B}}$ and a constant temperature $\mathcal{T}_{\mathrm{B}}$. Advection normal to the bed also has a major influence on stability. If the advection velocity is taken to vary linearly with depth and the bed is frozen, the effect of upward advection is to decrease stability and of downward advection to increase it. When the bed is temperate the effect of advection is more complex: downward advection increases stability but upward advection may increase or decrease it depending on the magnitude of the advection velocity.

\title{
DISCUSSION
}

W. F. Budd: Have you examined real situation values in your analysis to see the limits for which you can obtain stable solutions?

G. K. C. Clanke: I have just begun to do this and would like to satisfy myself that the numerical methods used to compute eigenvalues are sound and to test a range of flow laws before making generalizations about the region of stability.

\section{ICE SLIDING AND FRICTION EXPERIMENTS}

W. F. BudD

(Antarctic Division, Department of Science, 568 St Kilda Road, Melbourne, Victoria 3oo4, Australia)

Abstract. We are interested in studying the processes of sliding of ice over a variety of rock surfaces with the object of determining an empirical relation for the basal shear stress appropriate for glaciers. The variables to be considered include: normal stress $\mathcal{N}$, shear stress $\tau$, surface roughness $r$, sliding velocity $V$, temperature $\theta$, water at the interface, and the presence of debris. The roughness is considered to be a function of two variables; the scale or wavelength $\lambda$, and the shape or slope of the roughness $a / \lambda$, where $a$ is the amplitude of the variations of that scale.

The experiments are to be carried out in three main phases viz:

(i) Limiting friction tests for no acceleration. For these the ice is placed on a slab and loaded with a normal stress, then the shear stress increased until acceleration occurs. So far the results suggest that for a given roughness the limiting shear stress is linearly related to the normal stress. Typical magnitudes for the limiting coefficient of friction are $\mu \approx 0.35$ for rough slabs. 
(ii) The second phase, now in progress, is for studying steady sliding under constant load. For this the ice is loaded on the slab with a normal stress and a shear stress well below the limiting value is applied. The motion over extended periods is then monitored. Sliding speeds comparable to real glacier sliding speeds are obtained.

(iii) For the third phase a constant velocity is applied and the shear stress is measured. A large horizontal wheel is driven to rotate about its centre for a wide range of constant speeds. A number of fixed rods are set like spokes above the wheel to hold the ice specimens. The ice cylinders are placed on the wheel, loaded with normal stress, and attached by spring balances to the spokes to measure the shear stress.

Surfaces of a variety of roughnesses are used. Temperatures near the melting point are of most interest, so large ice baths are used. Debris is found to increase the friction greatly. The melt water produced tends to be forced out from the interface. This is expected to simulate the flow of frictional melting under glaciers away from the high stress regions over bumps to the lower stress areas of the major channels. So far basal shear stresses of 1.5 bars have been achieved for ice near melting point. The experiments are being carried out by Peter Keage of the Antarctic Division.

\section{DISCUSSION}

T. J. Hughes: Would you please describe the geometry of mounting ice-core slices on your wheel for creep and sliding studies?

W. F. BudD: The wheel described is just for sliding experiments. The ice-core specimens are vertical cylinders resting on a flat horizontal wheel base and are loaded at the top. They are held to a spoke by a spring balance while they rest and slide on the wheel as it rotates at a constant speed. Thus for a set load and sliding speed the shear stress can be measured. 